\title{
The Effect of Effleurage Massage Therapy on Symptoms of Osteoarthritis in Elderly Women with Osteoarthritis: A Cross- Clinical Trial
}

\author{
Zohreh Abbasi ${ }^{1 *}$, Mahbobeh Hakimi Najaf Abadi ${ }^{2}$, Reza Ganji ${ }^{3}$, Raheleh Asali ${ }^{1}$ Seyed Hamid Nabavi ${ }^{4}$, \\ Seyedeh Mahboobeh Rezaeean ${ }^{1}$, Salar Poorbarat ${ }^{5}$
}

${ }^{1}$ Department of Midwifery, Faculty of Medicine, North Khorasan University of Medical Sciences, Bojnurd, Iran; ${ }^{2}$ Master of Geriatric Nursing, North Khorasan University of Medical Sciences, Imam Hassan Hospital, Bojnurd, Iran; ${ }^{3}$ Department of Orthopedic Surgery, North Khorasan University of Medical Sciences, Bojnurd, Iran; ${ }^{4}$ Department of Public Health, School of Health, North Khorasan University of Medical Sciences, Bojnurd, Iran; ${ }^{5}$ Student Research Committee,, North Khorasan University of Medical Sciences, Bojnurd, Iran

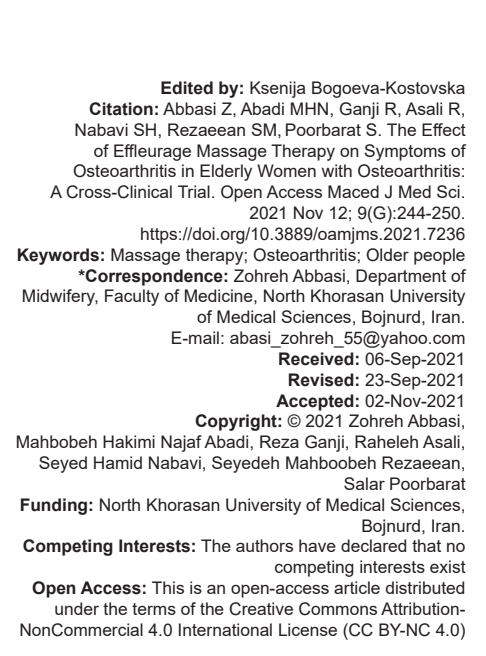

\begin{abstract}
BACKGROUND: Osteoarthritis is the main and most common cause of pain and disability in older people. It affects women 3.5 times more than men.

AIM: Therefore, the present study aimed to determine the effect of effleurage massage therapy on symptoms of osteoarthritis in elderly women with osteoarthritis.

MATERIALS AND METHODS: The present study was a cross-sectional clinical trial with a control group on 58 elderly women with mild to moderate osteoarthritis in 2018. At the first stage of the study, we give a massage on each knee every day for $15 \mathrm{~min}$ a week by the Effleurage method with standard treatment (100 mg diclofenac) and the control group only received standard treatment. At the second stage, the intervention was shifted in groups according to the study type after 3 weeks of washout. The research instrument was a native questionnaire (The Knee Injury and Osteoarthritis Outcome Score [KOOS]). We analyzed data using the SPSS20.

RESULTS: The research results indicated that knee massage techniques could reduce the severity of symptoms at both stages of the study in the intervention group. At the first stage, the severity of symptoms in Group A (intervention group) significantly decreased after the intervention compared to the pre-intervention and also decreased after washout in Group B (intervention group) after the intervention compared to the pre-intervention $(p<0.0001)$.

CONCLUSION: Given the effect of massage on reducing symptoms of knee osteoarthritis (morning dryness, stiffness, and swelling,) in elderly women with osteoarthritis, massage therapy is recommended as an effective intervention to reduce symptoms of knee osteoarthritis in these patients.
\end{abstract}

\section{Introduction}

Aging is an inevitable period of life, and every person will face this stage in the evolutionary process of life [1]. It is associated with physiological changes in the body, lower function and independence, higher disease and disability, and dysfunction of various organs of the body [2]. Natural and physiological changes differentiate aging, cause changes in health needs, and increase the burden of diseases and health costs [3]. In recent years, we have faced a growing trend of aging around the world so that the percentage of the population over 60 years of age has almost doubled from $12 \%$ to $22 \%$ between 2015 and 2050 [4]. It is predicted that the older population of Iran will reach $21.7 \%$ by 2050 . After the UAE and Bahrain, Iran is the third country that is rapidly heading towards old age [5]. According to the latest census in Iran, women constitute $51 \%$, and men $49 \%$ of the elderly population in Iran [5]. Aging is associated with the spread of several diseases, including osteoarthritis that is the second most common chronic disease of old age. It is a classic age-related disorder [4]. Osteoarthritis is a major cause of the inability to perform daily activities, and the knee is a commonly involved joint [6]. Knee osteoarthritis is the most common joint disease in the human body, characterized by the destruction of hyaline cartilage. This disease is caused by the process of wear and tear that occurs with age and over time [7]. In the world, $40 \%$ of people over the age of 70 suffer from knee osteoarthritis. Arthritis, especially knee or hip joint arthritis, can affect the intensity of activities such as walking, climbing stairs, and using the bathroom [6]. The most common clinical symptoms of this disease include joint pain when moving, morning pain and stiffness of joints, stiffness of movements (limited joint movements), and dry joints, and patients occasionally say that the knee movement is accompanied by 
sounds [8]. The disease affects women 3.5 times more often than men, and the movement limitation of joints and the resulting pain limit patients to do many things, leading to disability, especially in the elderly women [9].

There is no definitive treatment for this disease and treatment strategies reduce the progression of the disease, control pain, reduction of joint dryness, maintaining functional capacity, and ultimately improving the patients' quality of life [10]. Common drug therapies include drugs, which quickly relieve symptoms such as acetaminophen, and nonsteroidal anti-inflammatory drugs (NSAIDs), which are slow in relieving symptoms such as chondroitin sulfate and Piascledine, and structurally effective drugs that are likely to reduce the rate of structural changes in osteoarthritis. Topical use of NSAIDs such as diclofenac controls pain and inflammation and causes side effects such as headache, dizziness, skin rash, edema, and kidney and liver damages. These side effects limit its use [11]. Surgical treatments using a prosthesis or osteochondral transplant will last for up to 15 years at best. Following knee arthroplasty, patients are exposed to risks such as deep vein thrombosis and venous thromboembolism [12]. Other treatments include alternative and complementary therapies, including acupuncture, herbal medicine, homeopathy, magnetic fields, spiritual therapies, supplements, and massage therapy [13]. Massage therapy is an ancient method of therapy that is now gaining popularity as a part of the alternative and complementary medicine movement [14]. Bervoets et al. (2015) found that massage therapy in knee and shoulder osteoarthritis, in comparison with no treatment, improves pain and function in the short-term, but it has no advantage over the active treatment of osteoarthritis [15]. However, Adam I. Perlman et al. (2012) conducted a study titled "The appropriate time for massage therapy in knee osteoarthritis" and reported that massage therapy might improve pain and functions in patients, and a group, which was under massage therapy for more time, had a better therapeutic response to therapy [16]. Given the growing older population in the world, the high population of older women compared to older men in Iran, the need to improve the symptoms of osteoarthritis in older people, including morning dryness, stiffness, and swelling in the knee joint, and complications of treatment, the present study aimed to determine the effect of effleurage massage therapy on symptoms of osteoarthritis (morning dryness, stiffness, and swelling of the knee joint) in elderly women with osteoarthritis.

\section{Materials and Methods}

The present clinical trial study (IRCT20200811048370N1) was derived from a master thesis in Midwifery.The present two-group, single-blind cross-sectional clinical trial (the evaluator, who filled the questionnaires, was blinded to the study) had a purposive sampling method and examined 60-year-old women and older who visited the Rheumatology and Orthopedic Clinic of Bojnurd in the fall and winter of 2018 with a diagnosis of mild to moderate definite osteoarthritis by physicians using the knee X-ray and based on the Kellgren and Lawrence system. The research purpose, method, and confidentiality of information were first explained to the patients and written consent was obtained from the research units. Inclusion criteria: no disease-threatening joint disease, osteonecrosis, osteoporosis, rheumatoid arthritis, neuromuscular disease, no lower limb disease (no injection and knee surgery), no anticoagulant therapy or coagulation problem, no massage therapy during the past 3 months, no addiction, and no use of any pharmacological or non-pharmacological alternative treatments.

\section{Exclusion criteria}

Unwillingness to participate and cooperate in research and non-participation in two consecutive massage sessions or more were excluded from the study. Using the sample size formula $(Z a=1 / 96$, $Z \beta=1 / 28, \mu 1 \pm \alpha 14 / 29 \pm 0 / 99, \mu 2 \pm \alpha 15 / 30 \pm 1 / 15)$, the sample size was 30 per group and a total of 60 individuals according to a $20 \%$ loss. With the exclusion of a person from the intervention group and a person from the control group due to the unwillingness to cooperate (Figure 1), the data analysis was finally performed on 58 individuals. Data collection tools included a global and localized questionnaire (The Knee Injury and Osteoarthritis Outcome Score (KOOS)) and a demographic information questionnaire. KOOS has 42 patient-centered questions that examined five concepts, namely, pain (9 questions), other symptoms (swelling, dryness, stiffness, etc.) of the disease (7 questions), daily activities (walking up and down the stairs, standing, bathing, etc.) (17 questions), sports and recreational activities (jumping, running, and spinning) (5 questions), and quality of life in terms of knee problems (4 questions). The study used questions about other symptoms of the disease (7 questions). There was no total score in the questionnaire. The scores of subscales were calculated separately. The total score of each subscale was divided by the maximum possible score of the subscale. It should be noted that a score of 100 is always considered as no problem and zero is the worst status in traditional orthopedics [17]. The questionnaire was localized by Solaleh Saraeipour in Iran in 2007 and its validity and reliability were

$$
n=\frac{\left(z_{1}-\frac{a}{2}+z_{1}-\beta\right)^{2}\left(\delta \frac{2}{1}+\delta \frac{2}{2}\right)}{\left(\mu 1-\mu_{2}\right)_{2}}
$$


confirmed with a Cronbach's alpha of $76 \%$ [17]. The reliability of the questionnaire was reconfirmed with Cronbach's alpha of $83 \%$. The demographic information questionnaire consists of explicit items (age, job, education level, place of residence, housing status, number of deliveries, insurance status, and marital status). The validity of the above-mentioned questionnaires was evaluated in terms of content so that the questionnaires were given to ten professors and faculty members of Bojnurd School of Nursing and Midwifery, and the final tool was used after considering the necessary suggestions and corrections and ensuring its comprehensiveness. The Knee Injury and Osteoarthritis Outcome Score (KOOS) was completed by the evaluator in the case of mild to moderate osteoarthritis. It should be noted that all questionnaires were completed during the study by an evaluator who was unaware of the research. To unify all participants and eliminate the effects of previous interventions and pharmacological and non-pharmacological treatments, all research units received standard treatment only for a week, and the use of other alternative therapies, which might affect the intervention, was eliminated. Then, the research units were divided into two groups of intervention or control based on the day of visiting the physician (even and odd day). After completing the questionnaires, the intervention group underwent individual massage and standard treatment (diclofenac $100 \mathrm{mg}$ daily) in the examination room. It should be noted that all massage techniques were performed by the researcher who had undergone training by the tutor in three sessions, and then a 20-min session for a final confirmation of the correctness of the massage by a traditional medicine specialist (Dr. Al Sheikh). Massage on each knee was performed using the Effleurage

$$
100-\frac{\text { Total score of the symptoms subscale }}{28} \times 100
$$



Figure 1: Consort flow diagram method by the researcher for 15 min every day for a week at a specific time (Effleurage is a type of Swedish massage performed as a sliding manipulation of superficial tissues with a slight pressure of two palms on the surface of the skin. It has a mild effect, but its main effect is on lymphatic and vascular regurgitation [18]). The amount of pressure was applied according to the tolerance of the research unit and the thinness of the elderly skin. During the massage process, research units were asked about this issue. Due to noninterference with other care and more patient comfort and homogenization, all interventions were performed in the afternoons from 16:00 to 19:00. Finally, the massage CD was delivered to the individuals, and their questions were answered. The control group received only standard treatment (diclofenac $100 \mathrm{mg}$ daily). After a week of intervention in both groups, the evaluator re-completed the KOOS questionnaire. Depending on the type of study (cross-clinical trial) and elimination of the possible effect of the intervention (given that the washout duration was three times higher than the halflife of the intervention and the half-life of the massage is a week) [13], [18], all research units rested for 3 weeks at this stage and no intervention was performed except for the standard treatment. The questionnaires were re-completed by the evaluator. The intervention and control were then replaced in a way that the control group became the intervention, and the intervention group became the control. According to the previous protocol, massage and control were performed on the target groups, and re-evaluation and filling the questionnaires were performed immediately after the last intervention session. We analyzed the data using the SPSS20. We used the Kolmogorov-Smirnov test to evaluate the normality of the distribution of quantitative variables. Descriptive statistics were used to describe quantitative and qualitative variables, and independent and paired t-tests were used to judge the effect of the intervention. The independent t-test was also used to evaluate the effects of time and treatment.

\section{Ethical considerations}

The present manuscript was taken from a master's thesis on old age, approved by the ethics committee of the North Khorasan University of Medical Sciences with a code (IR.NKUMS.REC.1397.043) and a Clinical trial code (IRCT20200811048370N1). Ethical consideration was explained to the participants.

\section{Results}

To evaluate the normality of the distribution of quantitative variables using the Kolmogorov-Smirnov test, the results indicated that all quantitative variables, 
Table 1: Mean and standard deviation of research units according to age, weight, and number of deliveries in two groups

\begin{tabular}{|c|c|c|c|c|}
\hline \multirow[t]{3}{*}{ Variable } & \multicolumn{4}{|l|}{ Group } \\
\hline & Group A & Group B & Average & Independent t-test result \\
\hline & Standard deviation \pm mean & Standard deviation \pm mean & & \\
\hline Age & $2.77 \pm 62.14$ & $22.3 \pm 62.40$ & $27.53 \pm 62.21$ & $p=0.993$ \\
\hline Weight & $39.6 \pm 65.77$ & $6.28 \pm 66.03$ & $39.6 \pm 65.77$ & $p=0.489$ \\
\hline Number of deliveries & $6.88 \pm 4.8$ & $1.75 \pm 5.5$ & $1.01 \pm 4.8$ & $p=0.458$ \\
\hline
\end{tabular}

except for age had a normal distribution. The results of age, weight, and the number of deliveries indicated that the mean age of the two groups was 62 years, mean weight was $65 \mathrm{~kg}$, and the mean number of deliveries was 4 to 5 , and there was no significant difference between the groups and they were homogeneous (Table 1).

In response to the research aim, "determining the mean scores of symptoms in older women in the two groups before and after the intervention and their comparison," the results of independent and paired t-test indicated that knee massage could decrease the severity of symptoms in Group A (the group that received massage and standard treatment) in the first phase of the study so that there was a significant difference between scores of symptoms before and after a week of massage. The scores of symptoms increased from $44.42 \pm 3.63$ to $66.23 \pm 1.17$ (the higher score meant the lower severity of symptoms) $(p<0.0001)$. In Group B (the group that received standard treatment), there was not any significant difference between symptom scores before and after a week of standard treatment $(p=0.086)$. In second phase of the study, we assessed the severity of symptoms after 3 weeks of washout and replacement of groups (so that Group A only received standard treatment and Group B simultaneously received standard treatment and massage), and the results indicated that mean scores of symptoms in the two Groups A and B were not statistically significant different based on the independent t-test and comparison of mean differences first before the second stage intervention (final washout), but after replacing the groups and performing the intervention (the Group $A$ only received the standard treatment and Group B simultaneously received the standard treatment and massage), the results of paired t-test indicated that knee massage decreased the severity of symptoms in Group B, and mean scores of symptoms increased from $44.46 \pm 0.48$ to $66.23 \pm 11.40$, so that there was a significant difference between scores of symptoms before and after a week of intervention (massage and standard treatment) $(p<0.0001)$, but there was not any significant difference between scores of symptoms before and after a week of standard treatment in Group A (that received standard treatment), $(p=0.569)$ (Table 2).

In calculating the effect of treatment and the difference between mean scores of variables on the first 7 days (first phase of the study) with a mean score of the second 7 days (second phase of the study), shown by DifT1, the independent t-test indicated that the effect of treatment was significant for the rate of symptoms and the components had a more favorable status in Group A ( $p<0.0001)$. About the effect of time, the difference between the mean scores of the variables on the second 7 days and the mean scores of the first 7 days, shown with DifT2, the independent t-test results indicated that the effect of time for the rate of symptoms was significant in the two groups, indicating the difference in turn of each treatment group $(p=0.030)($ Table 3$)$.

\section{Discussion}

The rate of symptoms based on the KOSS test refers to the morning dryness, stiffness, and swelling. The present results indicated that performing massage techniques for a week could improve symptoms of osteoarthritis (morning dryness, stiffness, and swelling) and the difference between the mean scores of massage-receiving groups was significant before and after replacement. In other words, the simultaneous use of massage and standard treatment (diclofenac) caused a greater reduction in the severity of symptoms in older women than in the control group, indicating the synergistic effect of both pharmacological and non-pharmacological treatments together so that the alternative medicine can be used simultaneously with

Table 2: Results of the effect of massage on the score of knee osteoarthritis symptoms in elderly women before and after the intervention in two groups

\begin{tabular}{|c|c|c|c|c|c|c|c|}
\hline \multirow[t]{3}{*}{ Variable } & \multirow[t]{3}{*}{ Group } & \multicolumn{3}{|l|}{ The first stage of the study } & \multicolumn{3}{|c|}{ The second stage of the study (after the end of 3 weeks Wash out) } \\
\hline & & Before the intervention & After the intervention & test results & Before the intervention & After the intervention & Paired t-test results \\
\hline & & Mean \pm standard deviation & Mean \pm standard deviation & & $\begin{array}{l}\text { Mean } \pm \text { standard } \\
\text { deviation }\end{array}$ & $\begin{array}{l}\text { Mean } \pm \text { standard } \\
\text { deviation }\end{array}$ & \\
\hline \multirow[t]{2}{*}{ Symptom score } & A & $3.63 \pm 44.42$ & $1.17 \pm 66.23$ & $\begin{array}{l}\mathrm{p}<0.0001 \\
\mathrm{t}=-4.16\end{array}$ & $10.77 \pm 54.46$ & $12.79 \pm 64.60$ & $\begin{array}{l}p=0.569 \\
T=-0.576\end{array}$ \\
\hline & B & $0.48 \pm 44.46$ & $14.9 \pm 41.25$ & $\begin{array}{l}p=0.086 \\
t=0.17\end{array}$ & $0.48 \pm 44.46$ & $11.40 \pm 66.23$ & $\begin{array}{l}p<0.0001 \\
t=9.72\end{array}$ \\
\hline $\begin{array}{l}\text { Mean difference } \\
\text { before and after }\end{array}$ & & $3.85 \pm-0.03$ & $2.73 \pm 24.96$ & & $0.29 \pm-1.1$ & $-19.37 \pm 99.39$ & \\
\hline Independent test & & & $\begin{array}{l}p<0.0001 \\
t=4.23\end{array}$ & & $\begin{array}{l}p=0.071 \\
t=7.23\end{array}$ & $\begin{array}{l}p<0.0001 \\
t=4.23\end{array}$ & \\
\hline
\end{tabular}


pharmacotherapy for more effectiveness according to national policies of Iran to reduce the severity of symptoms in patients with any disease. Therefore, massage or alternative medicine methods can improve pain by dilating superficial arteries, increasing blood flow, and excretion of waste [19], [20]. For the effect of alternative medicine, especially massage, there is an emphasis on frequent and continuous use; otherwise, we will see a reduction in the results [20]. This result was also confirmed by findings of the present study so that the score of symptom in Group A decreased after 3 weeks of washout $(45.46 \pm 10.77)$ compared to the $1^{\text {st }}$ week after the intervention $(66.23 \pm 1.17)$ (reduction of symptom score based on the KOSS test indicated an increase in the severity of symptoms). However, the increase in symptom severity was not as severe as at the beginning of the study.

Table 3: T-test results for therapeutic effect and time effect variables

\begin{tabular}{|c|c|c|c|}
\hline \multirow{3}{*}{ Variable } & \multicolumn{3}{|l|}{ Group } \\
\hline & Group A & Group B & Independent \\
\hline & $\overline{\text { Mean } \pm \text { Standard deviation }}$ & $\overline{\text { Mean } \pm \text { Standard deviation }}$ & t-test \\
\hline $\begin{array}{l}\text { The effect of } \\
\text { treatment }\end{array}$ & $61.9 \pm 4.01$ & $58.6 \pm 3.2$ & $p<0.0001$ \\
\hline DifT1 & & & $\begin{array}{l}t=7.407 \\
D F=27\end{array}$ \\
\hline $\begin{array}{l}\text { Time effect } \\
\text { DifT2 }\end{array}$ & $61.01 \pm 9.4$ & $58.32 \pm 26.6$ & $\begin{array}{l}p=0.030 \\
t=2.292\end{array}$ \\
\hline
\end{tabular}

The panel, which was held to review the available authoritative articles on different methods of physiotherapy for the treatment of knee osteoarthritis, reviewed 26 authoritative articles and provided guidelines for the treatment of the disease. According to the recommendations of the panel, exercise therapy, especially strengthening exercises and general physical activity relieved pain and improved performance. The use of exercise therapy alone and in combination with manual therapies such as massage has been recommended [21]. These results are consistent with various domestic and foreign studies, including studies of Mahmoudi (2015) [22], Atkiner (2009) [23], Adam Perlman (2014) [16], Jamshidifard and Mahjour (2015) [24], Tanoursaz et al. (2015) [25], Roshandel Hesari and Khodabandeh (2017) [26], Wentworth et al. (2009) [27], and Sherman et al. (2009) [28]. Given the type of study, that is, a cross-clinical trial, there was a difference in calculating the effect of time on the turn of each treatment group, and being in Group A led to the further improvement of the symptoms. Based on the results of the treatment effect, the rate of improvement of symptoms in Group A was greater than Group B probably because the research units of the study in Group A continued performing massage by themselves due to the reduction of osteoarthritis symptoms caused by massage. Therefore, it might justify a more favorable state of symptoms in the group that first received the massage. Based on the results of the treatment effect, the effectiveness of massage was higher than the standard treatment. The rate of symptoms, including joint dryness and swelling, was better in Group A than the Group B because a slight white discoloration was created on the skin due to the massage techniques and their effects on blood circulation and temporary and direct skin stimulation and due to the initial contraction of capillaries; hence, it affected the tissue and released histamine that initially led to redness and slight swelling due to the higher capillary permeability and accumulation of interstitial water and swelling, and gradually the swelling and redness decreased due to the higher tissue blood flow [29].

Mahmoudi (2015) studied the effect of massage and aromatherapy on osteoarthritis and reported that knee dryness immediately and a week after the massage was significantly different from the control group, but knee dryness was not significantly different between the two groups 4 weeks after massage [22], and the results of both stages were consistent with results of our study so that symptom score decreased in the massage-receiving group after 3 weeks of washout (Group A) (The lower score based on the KOSS test indicated an increase in the severity of symptoms). Furthermore, the scores of symptoms in the two groups were not statistically different after completion of washout and it emphasized the need for frequent and continuous use of alternative medicine, including massage [20].

Even though Adam Perlman (2012) did not examine the amount of knee dryness, stiffness, and swelling after the massage, the range of joint motion was statistically different after massage [16]. Since the improvement of range of motion indicated improvement in motion position of the feet and thus the probability of foot joint softening and reduction of joint stiffness and dryness, it was indirectly consistent with the results of the present study and we can conclude that massage can be effective in improving symptoms of joint destruction due to osteoarthritis.

Joberg et al. (2015) reported that massage improved stiffness, function, and pain in patients [30], and their results were consistent with our research. In a study by Wentworth et al. (2009), they reported the improvement in pain and symptoms after massage [27] and their result was consistent with our results. Roshandel Hesari and Khodabandeh (2017) conducted a study titled "Comparison of the effects of isotonic exercises and massage therapy on pain severity and disability in men with chronic low back pain and reported that massage significantly reduced pain compared to isotonic exercises, but the two groups were not significantly different in the improvement of symptoms even though both groups reported an improvement in rates of symptoms at baseline [26]; however, given that their research participants were younger than the participants in the present study in terms of mean age, and they had a more favorable status in terms of joint destruction after osteoarthritis, and due to the difference in types of study and their implementation, the factors 
might cause the difference between results of studies by Roshandel Hesari and Khodabandeh with the present study. Brosseau et al. (2012) conducted a review study on people with chronic neck pain caused by trigger points and concluded that massage could be effective in reducing neck pain and range of motion [31]. It seems that the changes were due to effects of mechanical and hormonal factors caused by massage in addition to the effective communication between the massager and the massage recipient so that the communication could reduce anxiety and pain and had a greater effect in massage [20].

Even though the rate of symptoms was also improved in our study, due to the contradictory studies in this field, it seems that there is a need for a study with a larger statistical population. The massage technique performed by the researcher and the long-term cooperation of the research units were probably the reasons for our significant results because emotional communication was an effective factor during the massage that was ignored in other studies.

Like any study, the present study had some weaknesses and shortcomings. Even though we sought to make the data analyst unaware of the study, unfortunately, we could not make the massage recipient and the massager unaware of the method of the study, and it was probably a kind of bias. However, we sought to match all the research units in both groups and intervening factors, and all massage techniques were performed by a person to avoid any difference in the method of massage and even in the method of communication; hence, it was a strength of the present study.

\section{Conclusion}

The results of the present study indicated that performing massage techniques for a week could improve symptoms of osteoarthritis (morning dryness, stiffness, and swelling), and proper massage techniques could be effective in reducing the symptoms. Even though there are many techniques, which can be effective in reducing symptoms, manual techniques such as massage therapy and stretching were the most important reasons for the higher tendency towards this type of treatment due to their availability, low cost, patient interest, informality, lack of complications, and greater effectiveness in treatment.

\section{Acknowledgments}

The present manuscript was taken from a master's thesis on old age, approved by the ethics committee of the North Khorasan University of Medical Sciences with a code (IR.NKUMS.REC.1397.043) and a Clinical trial code (IRCT20200811048370N1). We are grateful to the research deputy of North Khorasan University of Medical Sciences, as well as all officials, especially Dr. Ghazi Saeidi (Rheumatologist), and Dr. Peyman Al-Sheikh (Chinese medicine specialist), as well as the research units for their great cooperation in the present research.

\section{References}

1. Ramtin S, Nikpeyma N. Investigating the happiness promotion strategies in Iranian older adults: A review article. J Gerontol. 2020;4(3):40-55. https://doi.org/10.29252/joge.4.3.40

2. Shokouhi F, Tavassoli E, Amiripour A, Kaviani F. Quality of life of elderly living in nursing homes in Shahrekord. Sci J Nurs Midwifery Paramed Fac. 2020;5(4):84-93.

3. Mirzaei M, Ghahfarokhi MS. Demography of elder population in Iran over the period 1956 to 2006. Salmand Iran J Ageing. 2007;2(3):326-31.

4. Abbaszadeh Z, Rashedi V, Amiri A, Haghighi A, Kianmehr N Effectiveness of exercise with a mobile application on pain of knee osteoarthritis: A randomized clinical trial. J Gerontol. 2019;3(3):1-8. https://doi.org/10.29252/joge.3.3.59

5. Mohammadi S, Charati MY, Mousavinasab SN. Factors affecting Iran's population aging, 2016. Mazandaran Univ Med Sci. 2017;27(155):71-8.

6. Mortazavi H. Pakniyat A, Ganji R, Armat M, Tabatabaeichehr M, Saadati $\mathrm{H}$. The effect of self-management education program on disability of elderly patients with knee osteoarthritis referring to elderly care clinic of Imam Reza (AS) treatment center in Shiraz, 2015-2106. J North Khorasan Univ Med Sci. 2017;8(3):461-70. https://doi.org/10.18869/acadpub.jnkums.8.3.461

7. Shahimoradi M, Mollahosseini M, Eghbali M, Khajepour $M$, Fathollahi MS. Comparing the effects of electroacupuncture, low level laser and celecouib tablet on treatment of the knee joint osteoarthritis: A randomized single-blinded clinical trial. J Rafsanjan Univ Med Sci. 2017;16(9):819-34.

8. Salehi-Sahlabadi A, Khani V, Varkaneh H, Rahmani J, Zarezadeh M, Khorshidi M, et al. Clinical effectiveness of glucosamine compared to Chondroitin sulfate, hyaluronic acid on relieving pain in people with osteoarthritis: A network metaanalysis. J Sabzevar Univ Med Sci. 2020;27(2):245-55.

9. Etesami AS, Zamani J, Zolaktaf V, Ghasemi G. Effectiveness of aquatic exercise therapy on the quality of life in women with knee osteoarthritis. Autumn. 2015;10(3):62-71.

10. Naesdal J, Brown K. NSAID-associated adverse effects and acid control aids to prevent them. Drug Saf. 2006;29(2):119-32. https://doi.org/10.2165/00002018-200629020-00002 PMid:16454539

11. Dehghan M, Abdoli-tafti A, Ganji F, Ghaedi R. Comparison the effects of ginger (Zingiber officinale) jelly and piroxicam jelly on pain of knee osteoarthritis. Sci J Kurdistan Univ Med Sci. 2018;23:8-17.

12. Galat DD, Scott C, Larson DR, Harrington JR, Hanssen AD, Clarke HD. Surgical treatment of early wound complications following primary total knee arthroplasty. J Bone Joint Surg. 2009;91(1):48-54. https://doi.org/10.2106/jbjs.g.01371

13. Baenes PM, Bloom B, Nahin RL. Complementary and alternative medicine use among adults and children: United 
States, 2007. Natl Health Stat Report. 2008;10(12):1-23. https:// doi.org/10.1037/e623942009-001

PMid:19361005

14. Susan A, Salva MT. A Massage Therapy Principle and Practice. Philadelphia, PA, London, New York: WB Saunders Company; 2015.

15. Bervoets DC, Luijsterburg PA, Alessie JJ, Buijs MJ, Verhagen AP. Massage therapy has short-term benefits for people with common musculoskeletal disorders compared to no treatment: A systematic review. J Physiother. 2015;61(3):106-16. https:// doi.org/10.1016/j.jphys.2015.05.018 PMid:26093806

16. Perlman Al, Ali A, Njike VY, Hom D, Davidi A, Gould-Fogerite S, et al. Massage therapy for osteoarthritis of the knee: A randomized dose-finding trial. PLoS One. 2012;7(2):e30248. https://doi.org/10.1371/journal.pone.0030248 PMid:22347369

17. Saraei-Pour S, Salavati M, Akhbari B, Kazem-Nezhad A. Translation and adaptation of knee injury and osteoarthritis outcome score (KOOS) in to Persian and testing Persian version reliability among Iranians with osteoarthritis. JREHAB. 2007;8(1):42-6. https://doi.org/10.1016/j.joca.2008.03.004

18. Goldstein S, Casanelia L. The techniques of Swedish massage. Found Massage. 2009;5(2):163.

19. Nasiri A, Mahmodi MA, Nobakht Z. Effect of aromatherapy massage with lavender essential oil on pain in patients with osteoarthritis of the knee: A randomized controlled clinical trial. Complement Ther Clin Pract. 2016;25:75-80. https://doi. org/10.1016/j.ctcp.2016.08.002

\section{PMid:27863613}

20. Abasi Z,Abedini Z,Fadaey A, Esmaili H. Effect of Back Massage on Pain Intensity During Frist Stage of Labor in Primiparous Woman in Bentolhoda Maternity in Bojnord City; 2005.

21. Nahayatbin $M$, Ghasem $M$, Rahimi $A$, Naeim $S$, Tabatabaee $S$. The effect of Tai chi exercise on the symptoms of osteoarthritis: Systematic review. J Rehab Med.2013;2(2):55-62.

22. Mahmoodi MA. Effect of Aromatherapy Massage with Lavender Essential Oil (Lavandula angustifolia) on Pain, Stiffness and Physical Function in Patients with Osteoarthritis of the Knee; 2015.
23. Atkins DV, Eichler DA. The effects of self-massage on osteoarthritis of the knee: A randomized, controlled trial. Int J Ther Massage Bodywork. 2013;6(1):4-14. https://doi. org/10.3822/ijtmb.v6i1.119

PMid:23482239

24. Mahjoor M, Javaheri AA, Ariamennes AS, Yazdi NK. Comparison of therapeutic exercises in water and massage therapy on severity of pain and disability in men with non-specific chronic low back pain. J Paramed Sci Rehabil. 2015;4(1):68-74.

25. Tanorzar S, Behpour NV, Chapri L. Effect of two methods of massage and exercise selective in water on spinal flexibility and pain and disability in depressed elderly women Dezful. J Shahrekord Univ Med Sci. 2017;19(5):26-38.

26. Hesari AR, Khodabandeh D. Comparison of isotonic exercises and massage therapy on severity of pain and disability in men with chronic low back pain. Paramed Sci Mil Health. 2012;12(2):21-8.

27. Wentworth LJ, Briese LJ, Timimi FK, Sanvick CL, Bartel DC Cutshall SM, et al. Massage therapy reduces tension, anxiety, and pain in patients awaiting invasive cardiovascular procedures. Prog Cardiovasc Nurs. 2009;24(4):155-61. https:// doi.org/10.1111/j.1751-7117.2009.00054.x PMid:20002340

28. Sherman KJ, Cherkin DC, Hawkes RJ, Miglioretti DL, Deyo RA. Randomized trial of therapeutic massage for chronic neck pain. Clin J Pain. 2009;25(3):233-8. https://doi.org/10.1097/ ajp.0b013e31818b7912 PMid:19333174

29. Abbasi Z, Abedian Z. How to Reduce Labor Pain with Massage? Mashhad: Zarih Aftab Cultural Artistic and Publishing Institute; 2009

30. Juberg $M$, Jerger KK, Allen KD, Dmitrieva NO, Keever $T$, Perlman Al. Pilot study of massage in veterans with knee osteoarthritis. J Altern Complement Med. 2015;21(6):333-8. https://doi.org/10.1089/acm.2014.0254 PMid:25966332

31. Brosseau L, Wells GA, Tugwell P, Casimiro L, Novikov M, Loew L, et al. Ottawa panel evidence-based clinical practice guidelines on therapeutic massage for neck pain. J Bodywork Mov Ther 2012;16(3):300-25. https://doi.org/10.1016/j.jbmt.2012.04.001 PMid:22703740 